\title{
Originalarbeiten
}

\section{Reduzierung des Organohalogen-Gehaltes beim gefütterten Aal (Anguilla anguilla L.)}

\section{durch Hälterung in Wasser hoher Qualität}

\author{
G. Koss ${ }^{*}$, M. Schebek, M. Losekam, W. Adler, E. Schüler \\ Institut für Toxikologie und Pharmakologie der Philipps-Universität Marburg, Pilgrimstein 2, D-W-3550 Marburg \\ " Behörde für Arbeit, Gesundheit und Soziales der Freien und Hansestadt Hamburg, Tesdorpfstraße 8, D-W-2000 Hamburg 13
}

Zusammenfassung. Unter kontrollierten Bedingungen wurde an Aalen aus der Lahn die Elimination der in ihrem Gewebe angereicherten Organohalogen-Verbindungen $\alpha-\mathrm{HCH}, \mathrm{HCB}$, DDT und PCB untersucht. Sie wurden in Trinkwasseraquarien 16 Wochen gehältert und täglich gefüttert. In den ersten 4 Wochen der Hälterung kam es bei den Tieren zu einer geringfügigen Abnahme, in den letzten Wochen zu einer deutlichen Zunahme des Gewichtes.

Die unmittelbar nach dem Fang erfolgte analytische Erfassung der Organohalogene in Muskelfleischproben ergab Konzentrationen, welche die derzeit gültigen rechtlich normierten Grenzwerte nicht überschritten. Im Abstand von jeweils 4 Wochen wurden die Aale auf ihren Gehalt an Organohalogenen untersucht. Dabei wurde insbesondere in den letzten Hälterungswochen eine deutliche Abnahme der Organohalogen-Konzentration festgestellt. Die ermittelten Eliminationshalbwertzeiten der Substanzen lagen zwischen 9 und 23 Wochen.

Die Untersuchung zeigt, daß die Hälterung von Aalen unter den gewählten Bedingungen eine deutliche Reduzierung der in ihrem Gewebe angereicherten Organohalogene bewirkt.

\section{Abstract.}

Reduction of $\alpha-\mathrm{HCH}, \mathrm{HCB}, \mathrm{DDT}$, and PCB in Eel (Anguilla anguilla L.) Kept in Clean Vater.

Eel from the Lahn river was tested for its ability to eliminate $\alpha-\mathrm{HCH}, \mathrm{HCB}, \mathrm{DDT}$, and PCB. The eels were kept in tap water aquariums for 16 weeks. Food was provided daily. During the first 4 weeks the animals showed a slight decrease in body weight while towards the end of the study the body weight increased.

The analytical determination of the chlorinated compounds immediately after the animals were caught showed tissue concentrations below the German regulatory limits. Towards the end of the following 4 week-intervals an almost steady decline of the concentration was observed. The elimination half lives were calculated to 9-23 weeks.

\section{Einleitung und Problemstellung}

Die Hälterung von Aalen hat eine lange Tradition:

1. Sie wurde und wird angewendet, um „Mißgeschmack“ des Aalfleisches zu beseitigen (MANN 1960; TESCH
1983); (er läßt sich vermutlich auf Inhaltsstoffe aus dem Bodenbereich der Gewässer zurückführen, in dem sich der Aal zumeist aufhält).

2. Ein weiterer Aspekt könnte die Hälterung zur Routine werden lassen: Wegen des hohen Lipidanteils seiner Gewebe kann es zu extremer Anreicherung anthropogener Chemikalien im Aal kommen, als Folge der hohen Lipophilie dieser Substanzen. Bekannteste Beispiele sind die Isomeren des Hexachlorcyclohexans, DDT, Hexachlorbenzol, die PCB und die polychlorierten Dibenzodioxine und -furane (PCDD/PCDF).

Insofern bedeutet der Verzehr von Aalfleisch ein zusätzliches Gesundheitsrisiko. Zwar regulieren PHmV (1989) und SHmV (1988) den Verkehr (Handel) von Aalen, der allgemeine Verzicht auf den Verzehr wird dadurch jedoch nicht erreicht. In dieser Situation wäre eine Reduzierung der Schadstoffkonzentration durch Hälterung hilfreich. An eine Dekontamination, wie es gelegentlich dargestellt wird (BOISELlE und HILDEBRANDT 1989), ist bei Kenntnis der Substanzeigenschaften allerdings nicht zu denken (Koss et al., 1983; Koss, Manuskript in Vorbereitung).

In einer Studie an gehälterten Guppies wurden für hochchlorierte PCB Halbwertzeiten zwischen 2,5 und nahezu 7 Monaten festgestellt (BRUGGEMAN et al., 1984). Es ist davon auszugehen, daß beim Aal die Elimination von Organohalogen-Verbindungen in ähnlich großen Zeiträumen erfolgt. Daraus ergibt sich, daß selbst die gesamte Lebensspanne von Aalen für eine vollständige Elimination der Organohalogene, d.h. für eine Dekontamination, nicht ausreichen würde.

Diese Studie prüft, ob Aale, deren interne Belastung durch die Kontamination ihres Biotops zustandegekommen ist, die in ihrem Gewebe angereicherten Organohalogene wieder eliminieren, wenn sie unter Laborbedingungen in Wasser hoher Qualität gehältert und gefüttert werden.

Als Parameter wurden Organohalogene ausgewählt, die in Deutschland nicht mehr oder nur noch eingeschränkt zum Einsatz gelangen, die aber aufgrund ihrer Persistenz in der Umwelt nach wie vor eine weite Verbreitung haben.

* Korrespondenz: Priv.-Doz. Dr. G. Koss 


\section{Methoden}

\subsection{Tierhaltung}

Die Aale wurden aus der Lahn unterhalb Marburg/Cappel mit Reusen gefangen. Zu Transportzwecken wurden sie in einen Fischtransportbehälter überführt, der mit Wasser aus der Lahn gefüllt war. Anschließend wurden sie in Aquarien mit Trinkwasser (Leitungswasser) gehältert.

Die Aquarien befanden sich in einem geräuscharmen Raum mit indirektem Tageslicht und einer durchschnittlichen Temperatur von $20^{\circ} \mathrm{C}$. Zur Lichtseite waren die Aquarien mit Styroporplatten und oben mit Glasplatten abgedeckt, die eine Öffnung für Schläuche aufwiesen. Die Ganzglasaquarien hatten die Maße $50 \times 50 \times 100 \mathrm{~cm}(\mathrm{H} \times \mathrm{B} \times \mathrm{L})$. Die Wassermenge pro Aquarium betrug 160-180 Liter. Der Wasseraustausch war so geregelt, daß $0,51 / \mathrm{min}$ zu- bzw. abflossen. Dadurch war ein viermaliger Wasserwechsel pro Tag gewährleistet. Die täglich erfaßte Wassertemperatur in den Aquarien lag zwischen 13,5 und $16^{\circ} \mathrm{C}$. Mit handelsüblichen Aquarienpumpen wurde Raumluft mit einem Volumen von $1 \mathrm{l} / \mathrm{min}$ durch das Wasser geperlt. Als Unterschlupf für die Tiere wurden je Aquarium zwei V2aStahlwinkel mit einer Länge von $50 \mathrm{~cm}$ und einer Kantenlänge von $15 \mathrm{~cm}$ auf dem Aquariumboden plaziert. Einmal täglich wurden die festen Ausscheidungen der Tiere abgesaugt.

Als Futter wurden je Aal täglich 2 Tauwürmer (Lumbricus terrestris) mit einem durchschnittlichen Gewicht von $4 \mathrm{~g}$ pro Wurm (Fa. Grebenstein, Hannover) angeboten. Nicht angenommenes Futter wurde vor jeder weiteren Fütterung entfernt.

Für die Hälterungsdauer von 4-16 Wochen standen 4 Aquarien zur Verfügung, auf die dreimal sechs und einmal sieben Aale nach der Gewichtsbestimmung verteilt wurden. Um die Aale voneinander unterscheiden zu können, wurde ihnen mit einer Zange an bestimmter Stelle im Flossensaum eine Markierung eingestanzt. Innerhalb der ersten 5 Hälterungswochen starben 3 Aale. Sie wurden aus den Aquarien entfernt und nicht in die Untersuchungen miteinbezogen. Zum Ende jedes Hälterungsintervalls wurden jedem Aquarium 1 oder 2 Aale nach dem Zufallsprinzip mit einem Kescher entnommen und in der üblichen Weise betäubt. Nach Bestimmung von Gewicht und Länge wurden die Tiere durch Dekapitation getötet und Leber sowie Gewebeproben entnommen.

\subsection{Analytik}

Zur Prüfung der Aale auf Organohalogene wurde jedem Tier ungefähr in der Mitte zwischen Darmausgang und Schwanzende eine ca. 2 g schwere Muskelfleischprobe entnommen, von Haut befreit, gewogen, in flüssigem Stickstoff gefroren und in einem Mörser pulverisiert. Die Abtrennung von Wasser erfolgte durch Verreiben in Natriumsulfat. Die Isolierung von Fett und Organohalogenen erfolgte am Soxhlet mit einem $n$-Hexan/Aceton-Gemisch $(2: 1, \mathrm{~V} / \mathrm{V})$. Aliquote Teile des Extraktionsgemisches wurden für die Fettbestimmung und die Erfassung der Organohalogene verwendet. Die Fettbestimmung erfolgte gravimetrisch nach Abziehen des Extraktionsgemisches. Vor der Be- stimmung der Organohalogene wurden die im Extrakt enthaltenen Fette säulenchromatographisch (Florisil,60-100mesh,3Gew.-\%Wasser) abgetrennt. Alle Extraktionsmittel hatten den Reinheitsgrad „zur Rückstandsanalyse“. Cleanup-Hilfsmittel (Natriumsulfat, Florisil usw.) wurden vor der Verwendung auf evtl. vorhandene Kontaminationen durch die zu untersuchenden Organohalogene überprüft. Sämtliche Chemikalien wurden von Merck/Darmstadt bezogen. Detaillierte Angaben zur Methodik finden sich bei BECK und MATHAR (1985).

Um das Ausmaß der Extraktionsausbeute erfassen zu können, wurde die Wiederfindungsrate der Organohalogene bestimmt. Nach clean-up und Analyse zeigte sich, daß sich durchschnittlich mehr als $90 \%$ der zugesetzten Organohalogene wiederfinden ließen.

\section{Die Referenzsubstanzen}

$\alpha$-Hexachlorcyclohexan $(\alpha-\mathrm{HCH})$

Hexachlorbenzol (HCB)

$\mathrm{p}, \mathrm{p}^{\prime}$-Dichlordiphenyltrichlorethan ( $\mathrm{p}, \mathrm{p}^{\prime}-\mathrm{DDT}$ )

2,4,4'-Trichlorbiphenyl (PCB 28)

2,2',5,5'-Tetrachlorphenyl (PCB 52)

2,2'4,5,5'-Pentachlorbiphenyl (PCB 101)

$2,2^{\prime}, 3,4,4^{\prime}, 5^{\prime}$-Hexachlorbiphenyl (PCB 138)

$2,2^{\prime} 4,4^{\prime}, 5,5^{\prime}$-Hexachlorbiphenyl (PCB 153) und

$2,2^{\prime} 3,4,4$ ', 5, 5'-Heptachlorbiphenyl (PCB 180)

mit einer gaschromatographischen Reinheit von rund $99 \%$ wurden von Promochem/Wesel bezogen.

Die Bestimmung der Organohalogene erfolgte gaschromatographisch (Hewlett Packard Modell 5880 A) auf einer SE-54 fused silica-Kapillare $\{50 \mathrm{~m}$ Länge, $0,25 \mathrm{~mm}$ i.D., Macherey-Nagel). Trägergas war Helium mit einem Durchfluß von $1 \mathrm{ml} / \mathrm{min}$. Die Temperaturen von Injektor und ECD waren $200^{\circ} \mathrm{C}$ bzw. $250^{\circ} \mathrm{C}$. Für den Ofen wurde ein Temperaturprogramm mit der Anfangstemperatur von $120^{\circ} \mathrm{C}$ ( 1 min Dauer) und einer anschließenden Temperaturerhöhung von $1{ }^{\circ} \mathrm{C} / \mathrm{min}$ bis $180^{\circ} \mathrm{C}$ und von $2^{\circ} \mathrm{C} / \mathrm{min}$ bis $220^{\circ} \mathrm{C}$ gewählt. Die Probenaufgabe erfolgte splitlos.

\section{Ergebnisse}

\subsection{Biologische Daten}

Insgesamt wurden 22 Aale untersucht. Ihr durchschnittliches Körpergewicht, das unmittelbar vor dem Einsetzen in die Aquarien gemessen wurde, war $258 \mathrm{~g}\left( \pm 55 \mathrm{~g}^{1}\right)$. Die Extremwerte waren 106 und $361 \mathrm{~g}$. Einzeldaten sind nicht angegeben. Im Abstand von 4 Wochen wurde den Aquarien jeweils ein Aal entnommen und nochmals das Körpergewicht bestimmt. Anschließend wurden die Tiere betäubt und ihre Körperlänge gemessen. Die Länge der Tiere lag durchschnittlich über $50 \mathrm{~cm}$. Nachdem sie getötet worden waren, wurden Leber und Muskelfleisch entnommen und gewogen.

Das relative Lebergewicht war $1,8 \%$ bei den leichteren und $1,1 \%$ bei den schwereren Aalen. Bei der Präparation der Tiere für die weiteren Untersuchungen wurden makroskopisch erkennbare Ekto- und Endoparasiten nicht festgestellt.

${ }^{1}$ Standardabweichung 
In den ersten 8 Wochen kam es zu einer geringfügigen $\mathrm{Ab}$ nahme des Körpergewichtes. Im Verlauf der sich anschlieBenden 4 Wochen scheint eine Erholungsphase eingesetzt zu haben, die sich bis zum Ablauf der 16wöchigen Hälterung offensichtlich fortgesetzt hatte. Bis zu diesem Zeitpunkt hatte das Gewicht der Tiere um $11 \%$ zugenommen.

\subsection{Fettgehalt im Muskelfleisch}

Der durch Extraktion und Gravimetrie ermittelte relative Fettgehalt im Muskelfleisch der Aale lag zwischen 13 und $19 \%$.

\subsection{Analytische Daten}

Die in den Abb. 1 und 2 gezeigten Konzentrationen der 9 Organohalogene lassen eine starke Streuung erkennen $(\rightarrow A b b .1$ und 2). Sie beträgt in einzelnen Fällen mehr als eine Größenordnung. Bei der Auswertung der Ergebnisse war auffällig, daß manche Tiere durchgängig hohe Konzentrationen und andere Tiere durchgängig niedrige Konzentrationen an Organohalogenen aufwiesen. Eine Beziehung zum Einzelgewicht der Tiere oder zu deren Körperlänge war nicht zu erkennen, wohl aber zum Fettgehalt. Die Vermutung liegt nahe, daß die lokale Belastung des Lahnsediments oder der Beutetiere sowie besondere individuelle Verhaltensweisen oder Stoffwechselbesonderheiten die Höhe der Organohalogen-Konzentration bestimmt hatten.

Zur Frage, ob das Konzept der Studie einschließlich der auf 16 Wochen befristeten Hälterung geeignet war, eine Aussage zur Elimination der Organohalogene und ggf. über ihre Geschwindigkeit zu ermöglichen, wurden die für jeden Zeitpunkt bestimmten Meßwerte gemittelt und auf der Basis der Mittelwerte Regressionsgeraden berechnet (Verlaufsdaten $\rightarrow$ Tabelle 1). Entsprechende Graphiken zeigen die Abb. 1 und $2(\rightarrow A b b .1$ und 2). Damit ist belegt, daß alle 9 Organohalogene während der 16 wöchigen Hälterung einer deutlichen Elimination unterliegen. Wie die in Tabelle 1 angegebenen Korrelationskoeffizienten erkennen lassen, ist die signifikante Verknüpfung z.wischen der Konzentrationsabnahme und der Hälterungsdauer bei $\alpha$ $\mathrm{HCH}, \mathrm{p}, \mathrm{p}$-DDT, PCB138 und PCB180 deutlicher als bei den anderen Organohalogenen.

In Tabelle 2 sind die aus den Verlaufsdaten der Regressionsgeraden berechneten Halbwertzeiten der Organohalogene im Muskelfleisch angegeben $(\rightarrow$ Tabelle 2$)$. Sie liegen

Abb. 1: Abnahme der Konzentration von $\alpha-\mathrm{HCH}, \mathrm{HCB}$ und p,p'DDT im Muskelfleisch von Aalen im Verlauf einer 16wöchigen Hälterung in Aquarien mit täglich viermal gewechseltem Wasser (Leitungswasser). Die OrganohalogenKonzentration ist in $\mathrm{mg} / \mathrm{kg}$ Frischgewicht angegeben. Die eingezeichneten Symbole sind Einzelwerte. Sie entsprechen der Zahl der Aale, deren Muskelfleisch untersucht wurde.

Die für den Hälterungsbeginn (Zeitpunkt 0) angegebenen Werte repräsentieren die Gewebekonzentration an Organohalogenen ( ${ }_{n}$ Rückstände"), die sich durch den Aufenthalt der Tiere in der Lahn bis zum Zeitpunkt des Fanges ergeben hatte. Die über den gesamten Hälterungszeitraum eingetragene $\mathrm{Ge}$ rade (Regressionsgerade) wurde auf der Basis der Mittelwerte berechnet. Ihr Verlauf dokumentiert die durchschnitcliche Konzentrationsabnahme. zwischen 9 und 23 Wochen. Damit ist offensichtlich, daß Aale unter den Bedingungen hoher Wasserqualität in einem überschaubaren Zeitraum lipophile Organohalogene eliminieren.

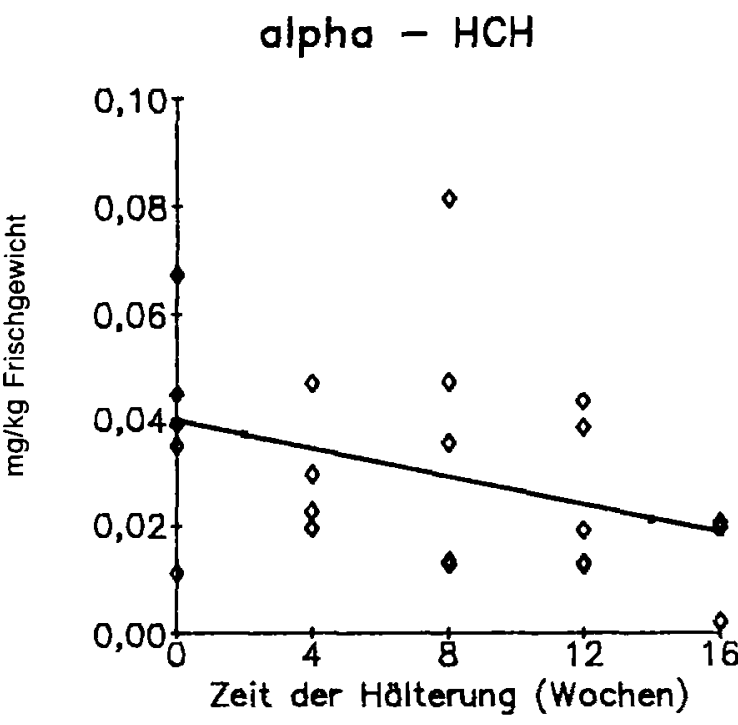

\section{HCB}
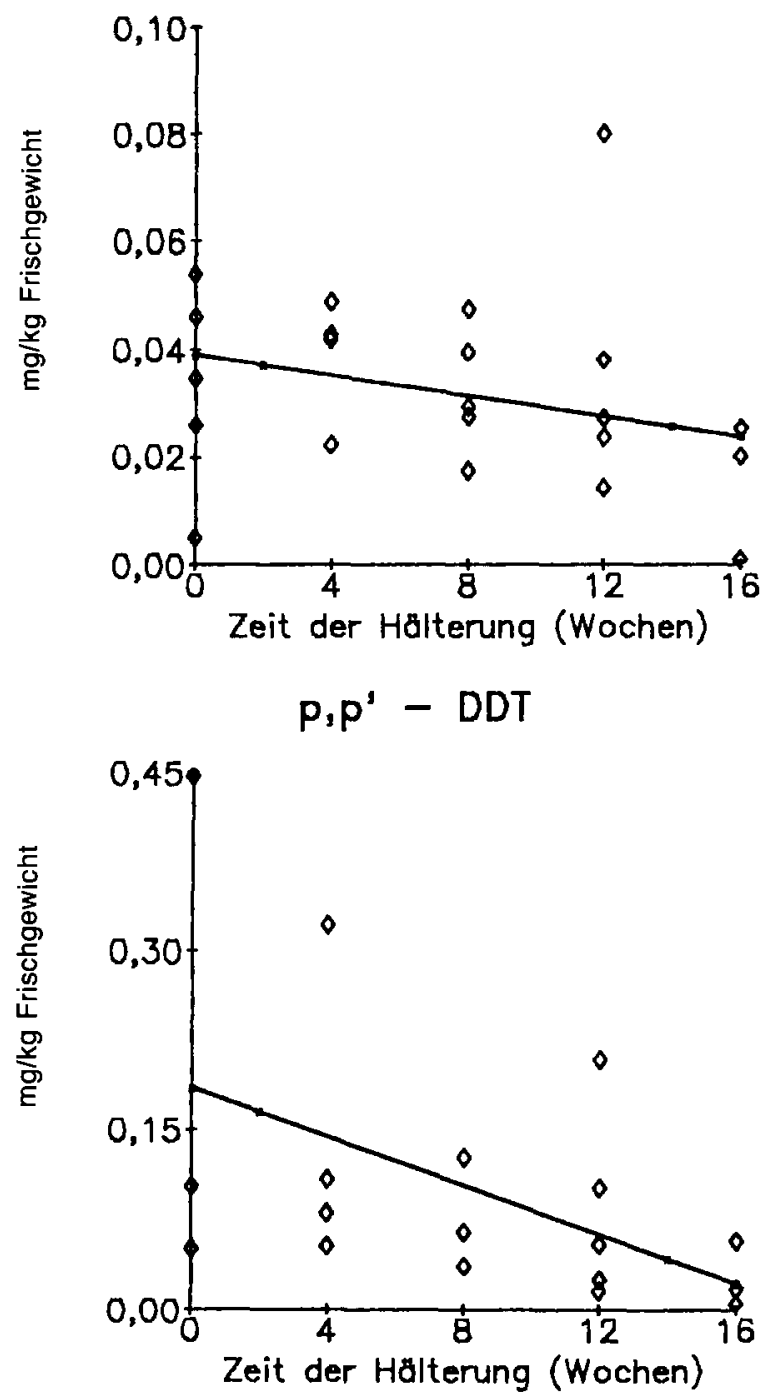
PCB 28
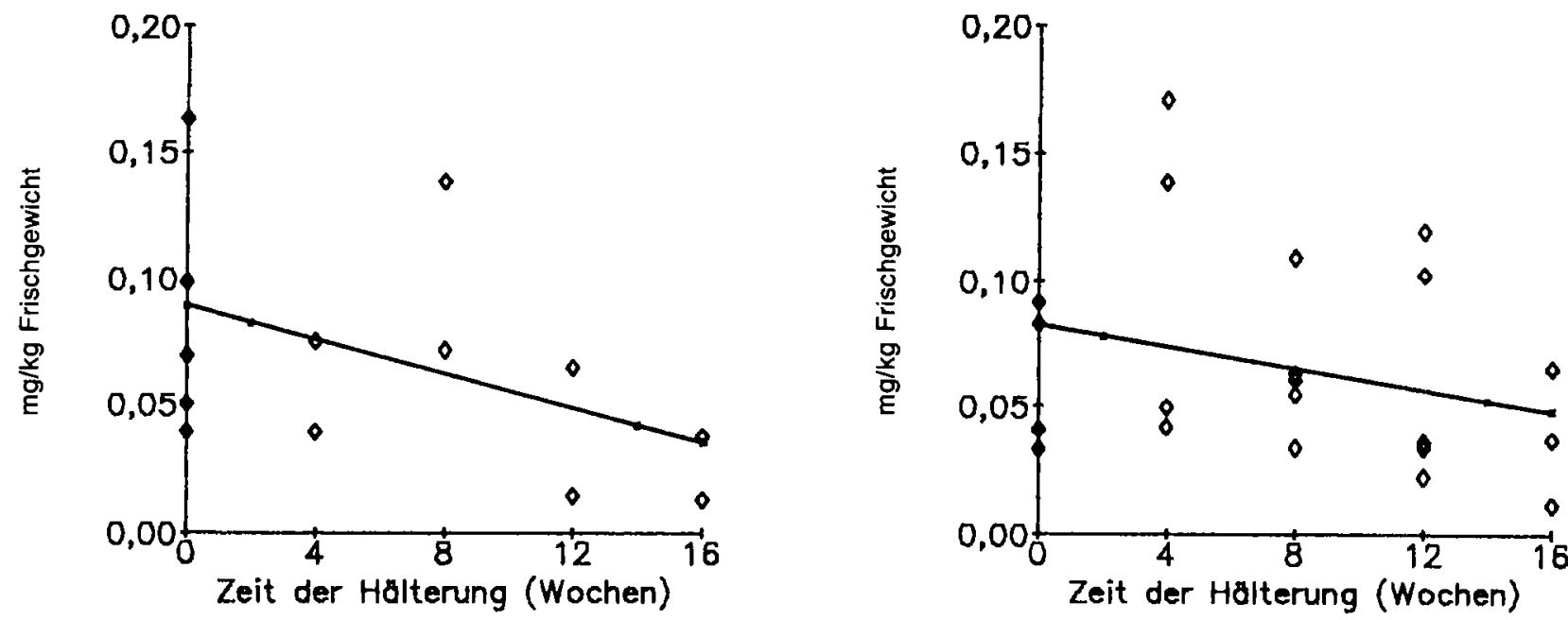

PCB 101
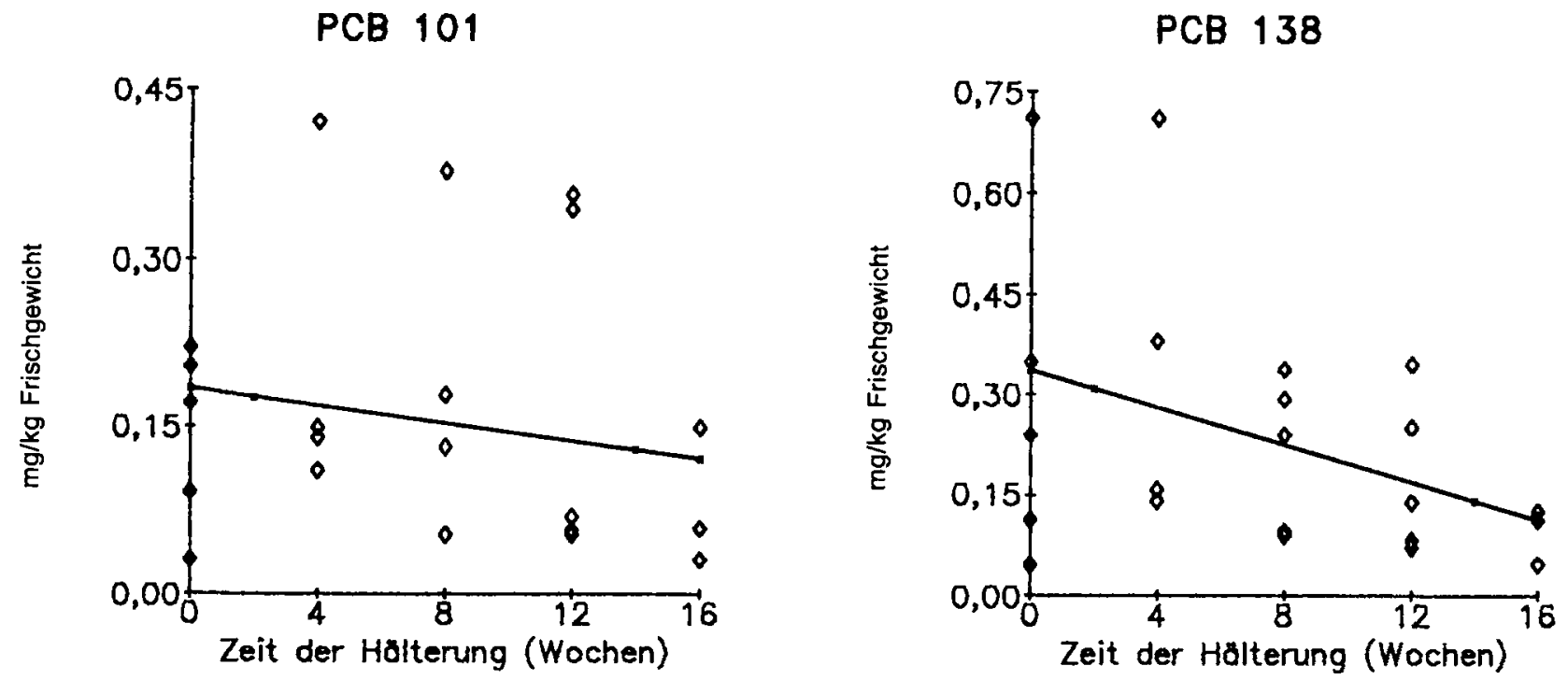

PCB 153
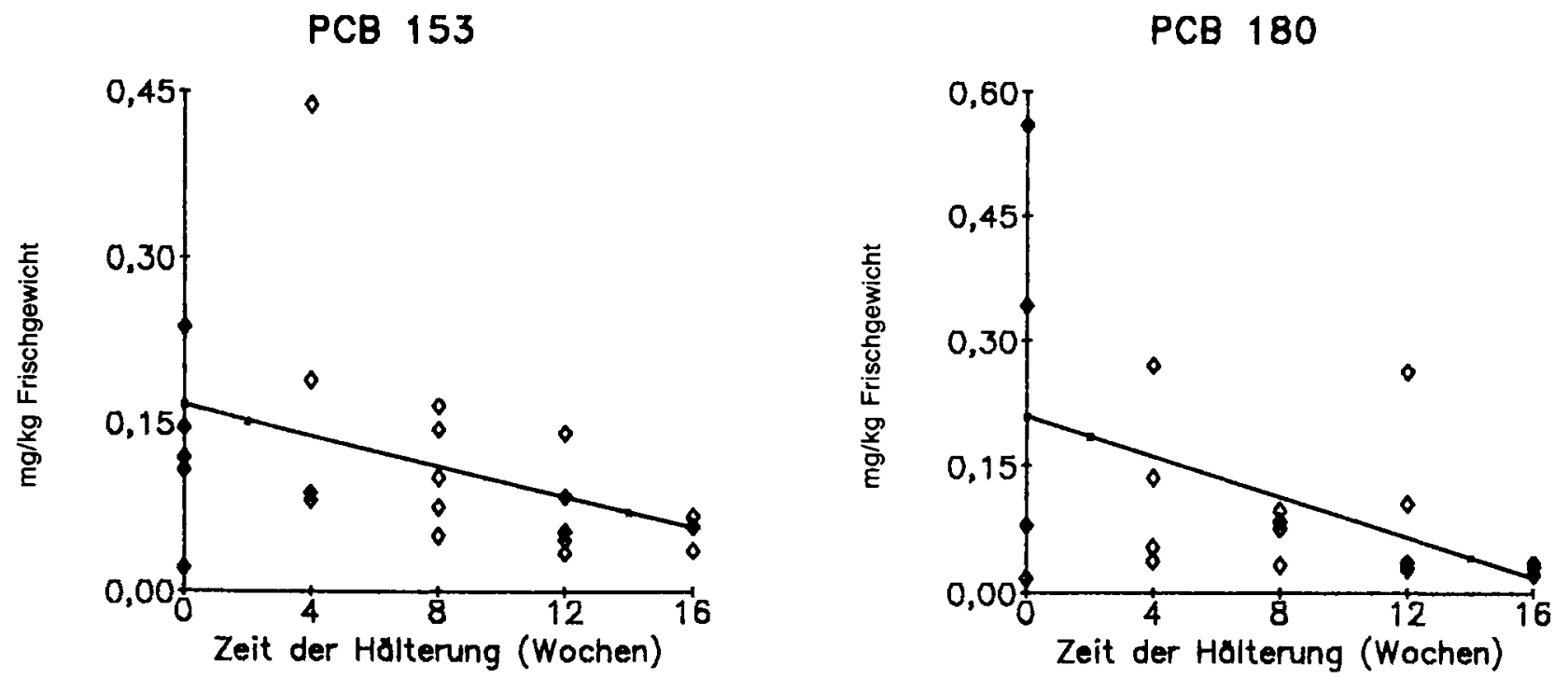

Abb. 2: Abnahme der Konzentration der 6 Indikator-Kongenere im Muskelfleisch von Aalen im Verlauf einer 16wöchigen Hälterung in Aquarien mit täglich viermal gewechseltem Wasser (Leitungswasser). Weitere Informationen vgl. Legende zu Abb. 1. 
Tabelle 1: Verlaufsdaten der Regressionsgeraden auf der Basis der Mittelwerte der zum jeweiligen Untersuchungszeitpunkt erfaßten einzelnen Organohalogen-Konzentrationen im Muskelfleisch (Frischgewicht) der Aale.

\begin{tabular}{c|c|c}
\hline $\begin{array}{c}\text { Organo- } \\
\text { halogen- } \\
\text { Verbindung }\end{array}$ & Verlaufsdaten & $\begin{array}{c}\text { Korrelations- } \\
\text { Koeffizient } \\
(r)\end{array}$ \\
\hline $\begin{array}{c}\alpha-H C H \\
\text { HCB }\end{array}$ & $Y=0,03954-0,00132 \times X$ & $-0,82700$ \\
p,p'-DDT & $Y=0,03879-0,00094 \times X$ & $-0,64346$ \\
PCB 28 & $Y=0,08932-0,00340 \times X$ & $-0,64469$ \\
PCB 52 & $Y=0,08264-0,00216 \times X$ & $-0,60869$ \\
PCB 101 & $Y=0,18426-0,00383 \times X$ & $-0,53205$ \\
PCB 153 & $X=0,16663-0,00681 \times X$ & $-0,75987$ \\
PCB 138 & $Y=0,33697-0,01411 \times X$ & $-0,90408$ \\
PCB 180 & $Y=0,20844-0,01187 \times X$ & $-0,89703$ \\
\hline
\end{tabular}

Tabelle 2: Halbwertzeit der Organohalogene im Muskelfleisch der Aale

\begin{tabular}{cc}
$\begin{array}{c}\text { Organohalogen- } \\
\text { Verbindung }\end{array}$ & $\begin{array}{c}\text { Halbwertzeita } \\
\text { in Wochen }\end{array}$ \\
\hline$\alpha-H C H$ & 14 \\
HCB & 21 \\
p,p'-DDT & 9 \\
PCB 28 & 13 \\
PCB 52 & 19 \\
PCB 101 & 23 \\
PCB 153 & 12 \\
PCB 138 & 12 \\
PCB 180 & 9 \\
\hline
\end{tabular}

a Die Halbwertzeit wurde aus dem Verlauf der Regressionsgeraden (vgl. Abb. 1 und 2, Tabelle 1) rechnerisch ermittelt.

\section{Diskussion}

\subsection{Toxikokinetik}

Die Datenlage zur Toxikokinetik unpolarer lipophiler Organohalogene sowohl im Säuger wie auch im Fisch berechtigte die Annahme, daß eine deutliche Verminderung des Gehaltes an Rückständen (HCB und p,p'-DDT) und Kontaminanten $(\alpha-\mathrm{HCH}$ und PCB) im Gewebe von Aalen in einem überschaubaren Zeitabschnitt erreichbar sein müsse. Bedingung wäre, 1. die Zufuhr der Substanzen möglichst vollständig zu unterbinden, 2. die Elimination der Chemikalien aus dem Gewebe, in dem sie angereichert wurden, zu begünstigen. Durch Verfüttern von Tauwürmern, die keinen nachweisbaren Gehalt an Organohalogenen aufwiesen (Ergebnisse nicht wiedergegeben), wurde die Invasion der untersuchten Organohalogene in das Gewebe der Aale fast vollständig unterbrochen. Eine nennenswerte Belastung der Fische während des Versuchs durch Organohalogene im Aquariumwasser, d.h. im Trinkwasser, wurde ausgeschlossen. Der zweite Teil der o.g. Bedingungen, die Elimination der Organohalogene, erfolgt hauptsächlich zusammen mit den Ausscheidungen über den Darm. Die Elimination über Haut und Kiemen in das Wasser spielt wegen der äußerst geringen Wasserlöslichkeit der Substanzen vermutlich eine eher vernachlässigbare Rolle.
Durch den täglichen mehrfachen Austausch des Hälterungswassers einschließlich der Entfernung der Ausscheidungen der Aale wurde eine Wiederaufnahme der ausgeschiedenen Organohalogene weitgehend verhindert. Ein Hinweis auf die Ausscheidung der Organohalogene in das Wasser war die unerwartet hohe Konzentration der Verbindungen im kondensierten Wasser an den Deckplatten der Aquarien (Ergebnisse nicht angegeben). Offensichtlich war ein Teil der von den Aalen eliminierten Organohalogene beim Verdampfen des Aquarienwassers aufgrund ihrer hohen Wasserdampfflüchtigkeit ebenfalls entwichen.

Für die Aufnahme der Organohalogene kommen neben der gastrointestinalen Resorption auch die Resorption über Kiemen und Haut in Betracht. Voraussetzung wäre allerdings, daß ein wesentlicher Teil der im Wasser befindlichen Substanzen in gelöster Form vorliegt. Wegen ihrer hohen Lipophilie und ihrer Fähigkeit zur Sorption wird allgemein davon ausgegangen, daß der weitaus überwiegende Teil der im Wasser enthaltenen Organohalogene an Partikel oder kolloidale organische Materialien adsorbiert ist (BRUGGEMAN et al., 1984). Die Resorption über Kiemen und Haut hat daher nur eine untergeordnete Bedeutung.

\subsection{Gewichtszunahme}

Die Gewichrszunahme, insbesondere in den letzten Hälterungswochen, zeigt, daß die gewählten Versuchsbedingungen größere Beeinträchtigungen der Aalentwicklung nicht herbeiführten und die Grenzen der physiologischen Adaption der Tiere nicht erreichten. Temperatur, Frischwasserversorgung, Belüftung und Lichtverhältnisse sowie die Besatzdichte in den Aquarien hatten offensichtlich keinen Einfluß auf die Aufnahme der angebotenen Tauwürmer. Das tägliche Angebot von durchschnittlich $8 \mathrm{~g}$ Futter pro Aal entspricht ungefähr der Erhaltungsfuttermenge (TESCH 1983). Insgesamt gesehen hat sich gezeigt, daß Streßfaktoren, die häufig zu Unverträglichkeiten zwischen den Tieren führen, weitgehend vermieden wurden.

\subsection{Halbwertzeiten}

Die ermittelten Halbwertzeiten sind nur von eingeschränkter Gültigkeit. Sie wären uneingeschränkt aussagekräftig, wenn die Elimination durch die, analytische Erfassung der Organohalogene am Anfang und Ende der Hälterung an demselben Tier geprüft worden wäre. Hierfür hätte es der operativen Probeentnahme beim Aal bedurft. Obwohl der Aal chirurgische Eingriffe offenbar gut übersteht (TESCH 1983), wurde davon abgesehen, um zusätzliche Streßfaktoren auszuschließen. Statt dessen wurden die Aale, die zur gleichen Zeit in einem engeren Bereich der Lahn gefangen worden sind, randomisiert in Gruppen zusammengefaßr und untersucht. Die beobachteten Halbwertzeiten sind daher nicht substanzspezifisch, sondern eher als Trendwerte für die gesamte Gruppe der untersuchten Organohalogene anzusehen.

Unter praxisnahen Bedingungen würde die in dieser Studie beobachtete längste Halbwertzeit, das sind 23 Wochen beim PCB 101, ein Orientierungswert für den zeitlichen Rahmen von Hälterungen sein. 


\subsection{Schlußfolgerungen}

Vor dem Hintergrund der hier beschriebenen, für die Aale optimalen Hälterungsbedingungen, sind die Ergebnisse der Studie besonders aussagekräftig. Die Auswahl der untersuchten Organohalogene erfolgte auf der Basis ihrer ökologischen und toxikologischen Bedeutung; sie deckt sich weitgehend mit den Untersuchungsparametern der Hessischen Landwirtschaftlichen Versuchsanstalt bei der Überprüfung der Schadstoffbelastung von Lahnfischen (HLVA 1990). In der eigenen Studie wurde statt des Lindans ( $\gamma$ $\mathrm{HCH}$ ) das $\alpha$-Isomere wegen seiner vergleichsweise höheren Persistenz in der Umwelt ausgewählt (DFG 1983).

\section{Danksagung}

Die Verfasser danken der Sportfischer-Vereinigung Marburg und Umgebung e.V. für die Unterstützung bei der Durchführung der Studie.

\section{Literatur}

BECK, H.; W. MATHAR: Analysenverfahren zur Bestimmung von ausgewählten PCB-Einzelkomponenten in Lebensmitteln. Bundesgesundheitsblatt 28, 1-12 (1985)

BoISELle, C.; G. HILDEBRANDT: Versuche zur ClKW-Dekontamination bei Aalen. Arch. Lebensmittelhyg. 40, 1-24 (1989)
Bruggeman, W.A.; A. Opperhuizen; A. Wijbenga; O. Hutzinger: Bioaccumulation of super-lipophilic chemicals in fish. Toxicol. Environmental Chem. 7, 173-189 (1984)

DFG 1983: Hexachlorcyclohexan als Schadstoff in Lebensmitteln. Deutsche Forschungsgemeinschaft, Forschungsbericht, Verlag Chemie, Weinheim 1983

HLVA 1990: Hessische Landwirtschaftliche Versuchsanstalt, Auswertung der Untersuchungsergebnisse von Lahnfischen, gefangen im Jahre 1989. Wiesbaden 1990

Koss, G.; S. Seubert; A. Seubert; J. Seidel; W. Koransky; H. IPPEN: Studies on the toxicology of hexachlorobenzene. V. Different phases of porphyria during and after treatment. Arch. Toxicol. 52, $13-22(1983)$

Koss, G.: 2,2',3,4,4',5,5'-Heptachlorbiphenyl. Manuskript in Vorbereitung

ManN, H.: Untersuchung über die Hälterung von Aalen. Arch. Fischereiwiss. 11, $145-151$ (1960)

PHmV 1989: Verordnung über Höchstmengen an Pflanzenschutz- und sonstigen Mitteln sowie anderen Schädlingsbekämpfungsmitteln in oder auf Lebensmitteln und Tabakerzeugnissen (Pflanzenschutzmittel-Höchstmengenverordnung - PHmV) vom 16. Okt. 1989, BGBl., 1989, Teil I, S. $1861-1908$

SHmV 1988: Verordnung über Höchstmengen an Schadstoffen in Lebensmitteln (Schadstoff-Höchstmengenverordnung - SHmV) vom 23. März 1988. BGBI., 1988, Teil I, S. $422-424$

Tesch, F.-W.: Der Aal. Biologie und Fischerei. Verlag Paul Parey, Hamburg/Berlin 1983, pp. 20

\section{Abbau von Linearen Alkylbenzolsulfonaten (LAS) unter Labor- und Freilandbedingungen mit einer neuen HPLC-Bestimmungsmethode}

\section{- Abschätzung des LAS-Gefährdungspotentials}

\section{J. Pflugmacher}

Dr. J. Pflugmacher, Biologische Bundesanstalt für Land- und Forstwirtschaft, Institut für Chemikalienprüfung, Königin-Luise-Straße 19, D-W-1000 Berlin 33

Zusammenfassung. Das Abbauverhalten von Linearen Alkylbenzolsulfonaten (LAS) im Boden wurde im Labor und im Freiland unter verschiedenen Bedingungen mit einer neu entwickelten Aufreinigungs- und Bestimmungsmethode auf Basis der HPLC untersucht, mit der sich Rückstände von LAS im Boden und Klärschlamm bis zu einer Nachweisgrenze von $0,01 \mathrm{mg} / \mathrm{kg}$ erfassen lassen. Die Wiederfindungsrate bei Zusatzversuchen im Boden betrug bei einer Konzentration von $0,02 \mathrm{mg} / \mathrm{kg} \mathrm{90,8} \pm 5,4 \%$. Unter aeroben nichtsterilen Bedingungen verläuft der Abbau am schnellsten (Halbwertzeiten: 5 - 7 Tage), unter anaeroben/anaeroben sterilen Bedingungen wesentlich langsamer (Halbwertzeiten: $40-120$ Tage).

\section{Abstract.}

Degradation of Linear Alkylbenzenesulfonates (LAS) in Soil Under Laboratory and Field Conditions with a New Detection Method. The degradation of linear alkylbenzenesulfonates (LAS) in soil was investigated under different laboratory and field conditions using a new HPLC method. The average recovery of LAS on a concentration level of $0.02 \mathrm{mg} / \mathrm{kg}$ in soil was $90.8 \pm 5.42 \%$. Detection limit $(S / N \geq 3)$ for the matrices soil and sewage sludge was $0.01 \mathrm{mg} / \mathrm{kg}$. Under aerobic non sterile conditions LAS decline rapidly (half lives: $5-7$ days); under anaerobic/anaerobic sterile conditions half lives were $40-120$ days.

\section{Einleitung und Problemstellung}

Lineare Alkylbenzolsulfonate (LAS) sind z.Zt. die am meisten produzierten anionischen oberflächenaktiven Substanzen. Sie werden als Bestandteil von Waschmitteln eingesetzt und gehören wegen ihres hohen Produktionsvolumens zu den mengenmäßig bedeutendsten organischen Chemikalien, die in Abwässern auftreten. Bei der Abwasserreinigung in den Klärwerken erfolgt eine Anreicherung im Klärschlamm (Durchschnitt $4 \mathrm{~g} / \mathrm{kg}$ Trockensubstanz nach [4]. Mit der Ausbringung von Klärschlamm auf landwirtschaftliche Nutzflächen gelangen sie in den Boden. Während über das Verhalten und die Abbaubarkeit im aquatischen Bereich vielfach publiziert wurde, sind für den terrestrischen Bereich nur wenige Daten verfügbar [10]. Kenntnisse über das Abbauverhalten im Boden sind wichtig, zum einen wegen der hohen Eintragsmenge, zum anderen wegen ihrer oberflächenaktiven Eigenschaft, die Löslichkeit anderer schwer wasserlöslicher Verbindungen zu erhöhen [12]. 